\title{
Anterior capsule contraction and intraocular lens dislocation in eyes with pseudoexfoliation syndrome
}

\author{
Hideyuki Hayashi, Ken Hayashi, Fuminori Nakao, Fumihiko Hayashi
}

\begin{abstract}
Aims-To examine the extent of anterior capsule contraction as well as intraocular lens (IOL) decentration and tilt following implant surgery in eyes with pseudoexfoliation syndrome (PE).

Methods-53 eyes from 53 patients with $P E$ and 53 control eyes from 53 age matched patients, undergoing phacoemulsification and implant surgery, were recruited. The anterior capsule opening area and the amounts of IOL decentration and tilt after undergoing continuous curvilinear capsulorhexis were measured using the Scheimpflug videophotography system at 1 week and $1,3,6,9$, and 12 months postoperatively.

Results-The mean area of the anterior capsule opening in the PE group was significantly smaller than that in the control group at 1 month postoperatively and later. The percentage reductions in the $\mathrm{PE}$ group were approximately $25 \%$, while they were less than $10 \%$ in the control group. The degree of IOL tilt was also larger in the PE group than in the control group. Five eyes $(9.4 \%)$ in the PE group underwent a neodymium:YAG laser anterior capsulotomy, but none in the control group underwent a capsulotomy.

Conclusions-The contraction of the anterior capsule opening was more extensive in the PE eyes than in the control eyes, thus resulting in a high Nd:YAG laser anterior capsulotomy rate. The IOL tilt was also greater in the PE eyes than in the control eyes.

(Br f Ophthalmol 1998;82:1429-1432)
\end{abstract}

Department of

School of Medicine,

Fukuoka University,

Fukuoka, Japan

H Hayashi

Hayashi Eye Hospital, Fukuoka, Japan

H Hayashi

K Hayashi

F Nakao

F Hayashi

Correspondence to: Hideyuki Hayashi, MD, Department of

Ophthalmology, School of Medicine, Fukuoka

University, Nanakuma,

Jonan-ku, Fukuoka 814-01, Japan.

Accepted for publication 23 July 1998 tion and fibrosis in the anterior capsule. Davison also reported eyes with pseudoexfoliation (PE) syndrome, which showed a marked constriction of the capsulorhexis opening and therefore required a neodymium $(\mathrm{Nd})$ :YAG laser anterior capsulotomy. ${ }^{9}$ Since the zonules have been demonstrated to be weak in PE syndrome, ${ }^{10-15}$ the anterior capsule contraction due to capsular shrinkage may be stronger than that observed in the healthy eyes.

Similarly, the dislocation of the implanted intraocular lens (IOL) is also considered to be greater in some pathological eyes than in healthy eyes. We found the decentration and tilt of the IOL in the eyes with retinitis pigmentosa to be larger than those in healthy eyes. ${ }^{8}$ In the PE eyes reported by Davison, the IOL also showed a marked displacement due to extensive capsule shrinkage. ${ }^{9}$

The objective of this study was to investigate the contraction of the anterior capsule opening and the extent of IOL decentration and tilt in PE syndrome. We also examined the incidence of the PE eyes which had to undergo an Nd:YAG laser anterior capsulotomy.

\section{Patients and methods}

Fifty three eyes from 53 patients with $\mathrm{PE}$ as well as 53 control eyes from 53 age matched patients, which were all scheduled to have phacoemulsification and IOL implant surgery between 1 June 1996 and 30 March 1997, were recruited for this study. Of the 53 eyes with PE, 24 eyes had pseudoexfoliative glaucoma, while 29 eyes did not. When we operated on the bilateral eyes, only the first operated eye was included in the analysis. For each eye from the patients with PE, a control eye from a non-PE patient was also selected within the 5 year age group. The preoperative exclusion criteria were (1) cataracts due to aetiologies other than age related changes, (2) a history of prior ocular surgery or inflammation, and (3) ocular pathologies such as diabetes mellitus and retinitis pigmentosa. All eyes had soft acrylic IOLs implanted with a $6.0 \mathrm{~mm}$ round optic (MA60BM; Alcon Surgical Inc, Fort Worth, TX, USA). Informed consent was obtained from all patients included in this study.

All surgeries were performed by a single surgeon $(\mathrm{KH})$. The surgical procedures have all been previously described. ${ }^{16}$ In brief, an approximately $3.5 \mathrm{~mm}$ scleral pocket incision was made for the soft acrylic IOL implantation. After the incision, continuous curvilinear capsulorhexis measuring about $5.5 \mathrm{~mm}$ in diameter was accomplished using a bent

Table 1 Patient characteristics

\begin{tabular}{llll}
\hline & PE group & Control group & p Value \\
\hline No of eyes & 53 & 53 & - \\
Age (SD) & $74.0(5.9)$ & $73.4(6.1)$ & $0.4389^{\star}$ \\
Sex (M/F) & $22 / 31$ & $18 / 35$ & $0.4228^{\star}$ \\
Left/right & $23 / 30$ & $22 / 31$ & $0.8442^{\star}$ \\
\hline
\end{tabular}

${ }^{\star}$ No statistical significance. 
Table 2 Mean area of the anterior capsule opening $\left(\mathrm{mm}^{2}\right)$ and the percentage reduction in the PE and control groups

\begin{tabular}{llll}
\hline & PE group & Control group & p Value \\
\hline Area $\left(\mathrm{mm}^{2}\right)(\mathrm{SD})$ & & & \\
$\quad 1$ week & $25.4(4.4)$ & $26.5(3.6)$ & $0.3185^{\star}$ \\
1 month & $21.1(6.3)$ & $25.1(4.7)$ & $0.0124 \dagger$ \\
3 months & $19.4(7.0)$ & $23.3(5.7)$ & $0.0219 \dagger$ \\
6 months & $20.1(7.2)$ & $24.2(5.7)$ & $0.0260 \dagger$ \\
9 months & $19.9(7.5)$ & $24.6(4.7)$ & $0.0143 \dagger$ \\
12 months & $19.7(7.8)$ & $24.8(4.8)$ & $0.0082 \dagger$ \\
Percentage reduction (SD) & & & \\
1 month & $17.5(16.4)$ & $5.6(8.7)$ & $0.0002 \dagger$ \\
3 months & $24.7(20.4)$ & $12.7(13.6)$ & $0.0066 \dagger$ \\
6 months & $23.9(20.4)$ & $9.4(13.4)$ & $0.0006 \dagger$ \\
9 months & $23.9(21.8)$ & $7.3(11.4)$ & $0.0005 \dagger$ \\
12 months & $22.3(19.8)$ & $6.7(11.8)$ & $0.0008 \dagger$ \\
\hline
\end{tabular}

^Not significant; †statistically significant.

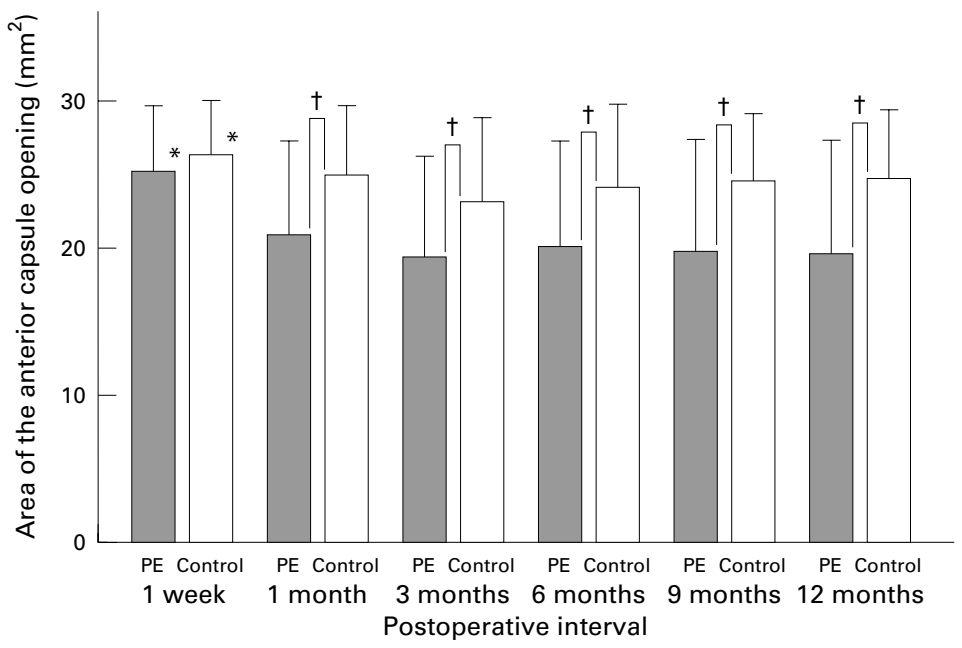

Figure 1 Comparison of the mean area of the anterior capsule opening between the PE and control groups. No statistical difference was observed in the capsulorhexis area at 1 week after surgery between the two groups. However, at 1 month and later, the mean area in the PE group was significantly smaller than that in the control group. ${ }^{\star} N o t$ statistically significant; tstatistically significant.

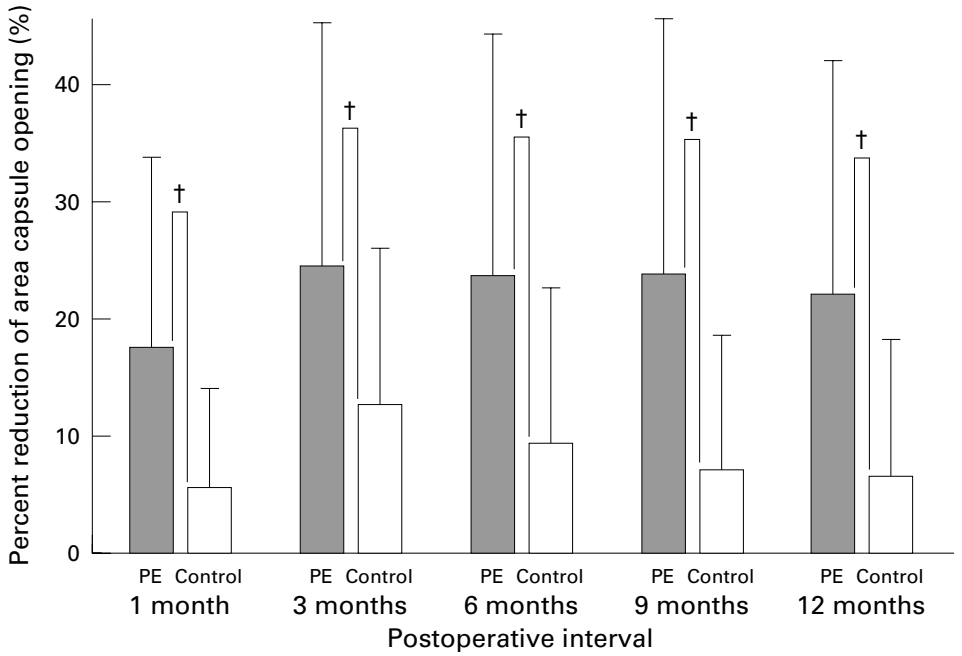

Figure 2 Comparison of the mean percentage reduction of the area of the anterior capsule opening between the PE and control groups. The percentage reduction in the area in the PE group was significantly greater than that in the control group throughout the follow up period. +Statistically significant.

needle. Following a hydrodissection, phacoemulsification of the nucleus and cortex aspiration were performed. The lens capsule was inflated with $1 \%$ sodium hyaluronate, and then the IOL was inserted into the capsular bag. At the end of surgery, the surgeon confirmed whether or not the IOLs were accu- rately placed in the capsular bag using a push and pull hook.

When the capsulorhexis opening was constricted to such an extent that the patient's vision was impaired, an anterior capsulotomy using an Nd:YAG laser was performed. ${ }^{9}{ }^{17} \mathrm{We}$ determined the basic criterion for the anterior capsulotomy to be a reduced opening area of less than $10 \mathrm{~mm}^{2}{ }^{8}$ When the capsulorhexis area became less than $10 \mathrm{~mm}^{2}$, the anterior capsule edge usually covered a portion of the pupillary area, thus leading to visual acuity loss or blurred vision. The surgical procedures of the Nd:YAG laser capsulotomy have all been previously described. ${ }^{8}$ In brief, an approximately $1 \mathrm{~mm}$ length of the anterior capsule edge with subcapsular fibrotic tissue was cut in four cross directions by the Nd:YAG laser burst.

Periodic examinations to quantify the anterior capsule opening area as well as the decentration length and tilt angle of the IOL were performed at 1 week, and 1, 3, 6, 9, and 12 months after surgery using the Anterior Eye Segment Analysis System (EAS 1000; Nidek Inc, Gamagori, Japan). The measurement methods to quantify the anterior capsule opening area, as well as the decentration length and tilt angle with the EAS-1000 system have all been described in previous studies. ${ }^{218}$ The best corrected visual acuity was also measured on decimal charts at all intervals. All measurements with the EAS-1000 system were carried out by three ophthalmic technicians, who were not informed of the purpose of this study.

Statistical analyses were performed to compare the differences in the measurement data between the PE and control groups using the Mann-Whitney U test. The categoric variables were compared using the $\chi^{2}$ test. Any differences showing a $\mathrm{p}$ value of less than 0.05 were considered to be statistically significant.

\section{Results}

Table 1 demonstrates the patient characteristics. The average patient age was 73.6 (SD 6.0) years old, with a range from 60 to 92 . No statistically significant differences were found between the PE and control groups regarding age, sex, or the ratio of the left and right eyes. In the first examination after full mydriasis at 1 week after surgery, all IOLs were confirmed to be placed in the capsular bag.

Table 2 summarises the mean area of the anterior capsule opening and the percentage reduction in the PE and control groups. The data are also displayed in Figures 1 and 2. The Mann-Whitney U test showed no significant difference in the mean capsulorhexis area at 1 week after surgery. However, at 1 month and later, the area in the PE group was significantly smaller than that in the control group. In addition, the percentage reduction in the PE group was also significantly greater than that in the control group. In the seven eyes $(13.2 \%)$ with $\mathrm{PE}$, the area markedly decreased to less than $10 \mathrm{~mm}^{2}$, but no eyes in the control group showed such a remarkable reduction. The visual acuity loss of these eyes from the best 
Table 3 Mean decentration length $(\mathrm{mm})$ and tilt angle $\left({ }^{\circ}\right)$ of the PE and control groups

\begin{tabular}{llll}
\hline & PE group & Control group & p Value \\
\hline $\begin{array}{l}\text { Decentration (mm) (SD) } \\
\text { 1 week }\end{array}$ & $0.304(0.203)$ & $0.295(0.236)$ & \\
1 month & $0.337(0.192)$ & $0.320(0.180)$ & $0.5782^{\star}$ \\
3 months & $0.339(0.202)$ & $0.312(0.161)$ & $0.7282^{\star}$ \\
6 months & $0.277(0.191)$ & $0.316(0.135)$ & $0.5869^{\star}$ \\
9 months & $0.290(0.146)$ & $0.313(0.167)$ & $0.0796^{\star}$ \\
12 months & $0.277(0.179)$ & $0.298(0.150)$ & $0.5689^{\star}$ \\
Tilt $\left({ }^{\circ}\right)(\mathrm{SD})$ & & & $0.3592^{\star}$ \\
1 week & $3.07(1.75)$ & $2.21(1.29)$ & $0.0114 \dagger$ \\
1 month & $3.44(2.01)$ & $2.35(1.29)$ & $0.0046 \dagger$ \\
3 months & $3.18(1.87)$ & $2.38(1.42)$ & $0.0140 \dagger$ \\
6 months & $3.02(1.52)$ & $2.41(1.44)$ & $0.0376 \dagger$ \\
9 months & $3.60(1.77)$ & $2.69(1.74)$ & $0.0063 \dagger$ \\
12 months & $3.66(2.12)$ & $2.77(1.97)$ & $0.0243 \dagger$ \\
\hline
\end{tabular}

^Not significant; †statistically significant.

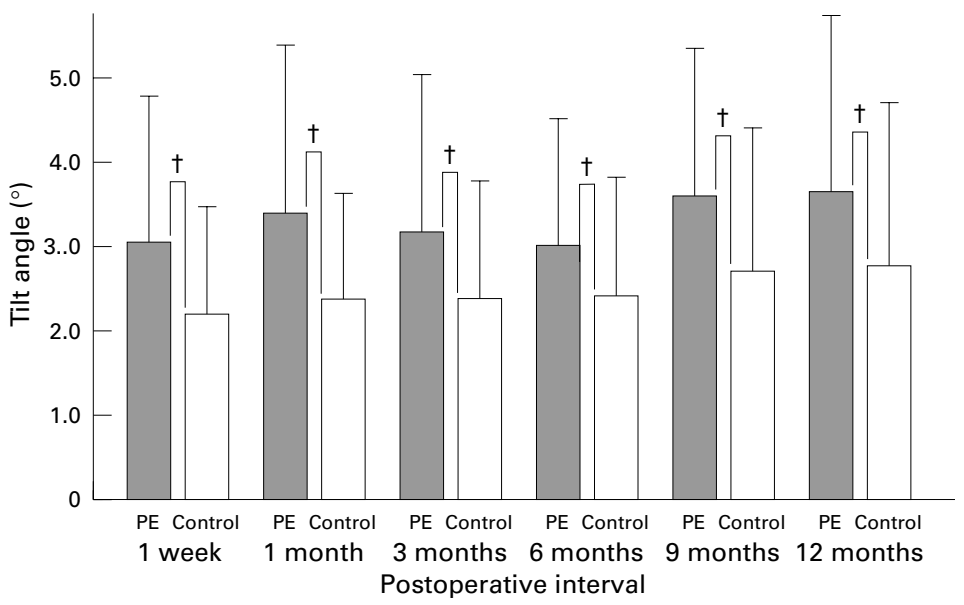

Figure 3 Comparison of the mean degree of the IOL tilt between the PE and control groups. The mean degree of the IOL tilt in the PE group was larger than that that in the control group throughout the follow up period. HStatistically significant.

postoperative acuity on the decimal charts was as follows; (1) two eyes, two lines or more, (2) three eyes, one line, (3) two eyes, no decrease.

Table 3 lists the mean IOL decentration length and tilt angle. The data of the tilt angle are also shown in Figure 3. The mean degree of the tilt in the PE group was significantly greater than that in the control group. However, the mean decentration length was almost the same between the two groups.

Five eyes $(9.4 \%)$ in the PE group underwent an anterior capsulotomy within 12 months postoperatively. The opening area of these eyes were all less than $10 \mathrm{~mm}^{2}$. The average interval between implant surgery and capsulotomy was 6.3 (3.8) months.

When comparing the eyes with and without glaucoma in the PE group, no statistical difference was observed regarding either the area reduction of the anterior capsule opening or the IOL decentration and tilt.

\section{Discussion}

Many kinds of pathologies are supposed to be predisposing factors for excessive capsule contraction after extracapsular cataract surgery. ${ }^{4-6}$ Davison previously reported on eyes with PE, which demonstrated a severe anterior capsule contraction with IOL displacement, and he attributed this complication to zonular weakness in PE syndrome. ${ }^{9}$

Our study clarified that the area reduction in the anterior capsule opening in the PE eyes was greater than that in the control eyes. The percentage reduction of the area in the PE group was approximately $25 \%$, while it was less than $10 \%$ in the control group. Furthermore, $13.2 \%$ of the PE eyes also showed an excessive reduction in the area to less than $10 \mathrm{~mm}^{2}$. These eyes all demonstrated either visual acuity loss or blurred vision. We therefore performed a radial anterior relaxing capsulotomy using an Nd:YAG laser for $9.4 \%$ of the PE eyes. Thus, the incidence of PE eyes which underwent a capsulotomy was considerably high compared with the other predisposing condition such as diabetes. ${ }^{7}$

The IOL tilt was also demonstrated to be greater in the PE eyes than in the healthy eyes, whereas the decentration was almost the same between the two groups We could not explain the reason why such an inconsistency exists regarding the results between the lens tilt and decentration. However, the greater the extent of the IOL tilt suggested that capsule shrinkage after extracapsular cataract surgery may be stronger in PE eyes than in the healthy eyes.

Many clinical and morphological studies demonstrated that the zonular apparatus in PE syndrome is friable and also occasionally causes spontaneous zonular disruption. ${ }^{10-15}$ Assia et al reported the stretching capability of the zonules to be weak in PE eyes. ${ }^{14}$ Furthermore, PE was also demonstrated to be a risk factor for developing zonular dialyses during extracapsular catract surgery. ${ }^{19}{ }^{20}$ As a result, an excessive degree of postoperative capsule shrinkage can be reasonably attributed to the zonular weakness in the PE eyes, which thus led to severe anterior capsule contraction and a greater extent of the IOL tilt. ${ }^{49}$

In this study, the contraction of the anterior capsule opening as well as the IOL decentration and tilt were similar between the eyes with and without glaucoma. These results thus suggest that the capsule shrinkage, which is possibly due to zonular weakness may not be related to the coexistence of glaucoma. Bartholomew also reported that long standing glaucoma plays no part in lens displacement. ${ }^{10}$

In conclusion, the contraction of the anterior capsule opening and IOL tilt was greater in the $\mathrm{PE}$ eyes than in the healthy eyes. Experienced surgeons are surely aware of the intraoperative risk of zonular dialysis when performing implant surgery for PE eyes. However, we must also be careful to find anterior capsule constriction in the early postoperative period. We usually perform an Nd:YAG laser anterior capsulotomy when the patient's vision is impaired. However, a careful follow up is necessary to confirm the long term safety of an anterior capsulotomy.

The authors have no proprietary interest in any of the materials described in this article.

Portions of this article were presented at the 24th Symposium on Cataract, IOL and Refractive Surgery from 18-22 April 1998, in San Diego, California, USA.

1 Joo CK, Shin JA, Kim JH. Capsular opening contraction after continuous curvilinear capsulorhexis. $\mathcal{f}$ Cataract Refract Surg 1996;22:585-90.

2 Hayashi K, Hayashi H, Nakao F, et al. Reduction in the area of the anterior capsule opening after polymethylmethacrof the anterior capsule opening after polymethylmethacr-
ylate, silicone, and soft acrylic intraocular lens implantation. Am f Ophthalmol 1997;123:441-7. 
3 Ursell PG, Spalton DJ, Pande MV. Anterior capsule stability in eyes with intraocular lenses made of poly(methyl
methacrylate), silicone, and Acrysof. 7 Cataract Refract Surg 1997;23:1532-8.

4 Hansen SO, Crandall AS, Olson RJ. Progressive constriction of the anterior capsular opening following intact capsulorhexis. F Cataract Refract Surg 1993;19:77-82.

5 Nishi O, Nishi K. intraocular lens encapsulation by shrinkage of the capsulorhexis opening. F Cataract Refract Surg 1993;19:544-5.

6 Namiki M, Yamamoto N, Tagami Y, et al. Risk factors for anterior capsule shrinkage in intraocular lens implantation. fpn f Clin Ophthalmol 1991;45:1828-31.

7 Hayashi H, Hayashi K, Nakao F, et al. Area reduction in the anterior capsule opening in eyes of diabetes mellitus patients. 7 Cataract Refract Surg 1998;24:1105-10

8 Hayashi K, Hayashi H, Matsuo K, et al. Anterior capsule contraction and intraocular lens dislocation following implant surgery in eyes with retinitis pigmentosa. Ophthalmology 1998;105:1239-43.

9 Davison JA. Capsule contraction syndrome. 7 Cataract Davison JA. Capsule contract

10 Bartholomew RS. Lens displacement associated with pseudocapsular exfoliation: a report on 19 cases in the southern Bantu. Br f Ophthalmol 1970;54:744-50.

11 Dark AJ, Streeten BW, Cornwall CC. Pseudoexfoliative disease of the lens: a study in electron microscopy and histochemistry. Br f Ophthalmol 1977;62:462-72.
12 Takei Y, Mizuno K. Electron-microscopic study of pseudoexfoliation of the lens. Graefes Arch Clin Exp Ophthalmol 1978;205:213-20.

13 Futa R, Furuyoshi N. Phakodonesis in capsular glaucoma: a clinical and electron microscopic study. Fpn f Ophthalmol 1989;33:311-17.

14 Assia EI, Apple DJ, Morgan RC, et al. The relationship between the stretching capability of the anterior capsule and zonules. Invest Ophthalmol Vis Sci 1991;32:2835-9.

15 Schlotzer-Schrehardt U, Naumann GOH. A histopathologic study of zonular instability in pseudoexfoliation logic study of zonular instability in pseudoe
syndrome. Am $\mathcal{f}$ Ophthalmol 1994;118:740-3.

16 Hayashi K, Hayashi H, Nakao F, et al. The correlationship between incision size and corneal shape changes in sutureless cataract surgery. Ophthalmology 1995;102:550-6.

17 Hurvitz LM. YAG anterior capsulectomy and lysis of posterior synechiae after cataract surgery. Ophthalmic Surg 1992; 23:103-7.

18 Hayashi K, Harada M, Hayashi H, et al. Decentration and tilt of polymethyl methacrylate, silicone, and acrylic soft intraocular lenses. Ophthalmology 1997;104:793-8.

19 Skuta GL, Parrish RK, Hodapp E, et al. Zonular dialysis during extracapsular cataract extraction in pseudoexfoliaduring extracapsular cataract extraction in pseud
tion syndrome. Arch Ophthalmol 1987;105:632-4.

20 Guzek JP, Holm M, Cotter JB, et al. Risk factors for intraoperative complications in 1000 extracapsular cataract cases. Ophthalmology 1987;94:461-6. 